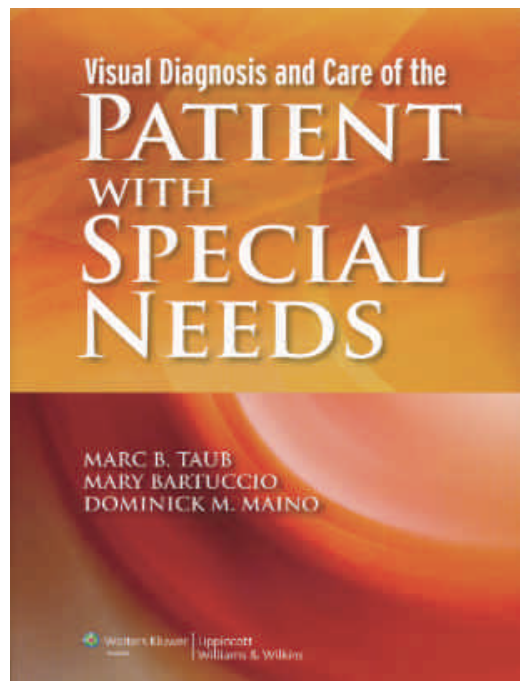

\section{BY CHERYL ZIMMER, OD, BSC}

The miracles of modern medicine have allowed premature infants a 90\% survival rate after only 27 weeks gestation, according to the March of Dimes.' Developmentally and intellectually delayed children now live to adulthood and seniors are living longer than ever imagined, often with multiple health issues. As optometrists, primary health care professionals, we have the responsibility to provide vision care to everyone, and we must be prepared for that endeavour. Visual Diagnosis and Care of the Patient with Special Needs written by Marc B. Taub, Mary Bartuccio and Dominick M. Maino, all doctors of optometry, is the essential resource for taking care of special populations.

The populations in this book include those that may be born with a disability or syndrome as a result of problems during gestation or genetic mutations, such as those with cerebral palsy, Down syndrome, fragile $X$ syndrome as well as intellectual disabilities of unknown origins, and those special populations that have acquired illnesses such as brain injury from disease, accident or stroke, psychiatric disorders and neurodegenerative diseases. Each condition is discussed from a systemic standpoint and oculovisual anomalies and their clinical implications are addressed.

\title{
Visual Diagnosis and Care of the Patient with Special Needs
}

The management and treatment of those with autism spectrum disorders, attention deficit hyperactivity disorder and learning disabilities are also discussed at length. These conditions are more readily diagnosed than ever before and more prevalent in the class room and the primary care optometrist's examination room. The Canadian Association of Optometrists, Eye Health Month's slogan for 2012 was Look, See, Learn. This applies to all children, and this book equips the optometrist to better deal with those for whom vision and learning are not straightforward.

Some visual issues affecting these populations include refractive errors, strabismus, amblyopia and visual field defects, as well as oculomotor dysfunctions. Computers have provided patients with better access to visual rehabilitation, treatment and enhancement. These techniques and procedures are addressed in detail for both in-office assessment and home use.

One other area of interest discussed at length is the visual processing issues that special needs populations encounter that cannot be treated with traditional spectacle therapy. The book introduces additional procedures not commonly used during the conventional comprehensive assessment of the visual system, but necessary for the assessment of the non-verbal patient or those with multiple system delay.

One of the most intriguing chapters outlines the optometric management of functional vision disorders. Treatment includes the use of spectacles and prisms to relieve stress on the visual system and improve binocular performance. In conjunction, occlusion and vision therapy may also be implemented. Case examples are used to illustrate these management techniques. The neurological basis for vision therapy is also discussed in this chapter, stressing the integration of vision with other sensory inputs and how these interwoven systems play such a vital role in a person's localization and orientation in the world.

The authors stress a multi-disciplinary approach where the patient's needs are assessed and treated by a variety of specializations including, but not limited, to their physician, neurologist, ophthalmologist and optometrist as well as adjunct health care workers such as chiropractors, occupational therapists, social workers and dieticians. The objective is to reduce the total load of systemic and visual assault on the body experienced by special needs patients with many of these debilitating syndromes. Enhanced communication between the disciplines is in the best interest of the patient.

The Visual Diagnosis and Care of the Patient with Special Needs may be read from cover to cover, providing the reader with a wealth of incredible and pertinent information, or used as a reference manual in the primary care optometry office. The authors take into account that the readers of this publication are well versed in the procedures used during a routine eye examination, but they expand the reader's knowledge base and apply these diagnostic and treatment techniques to those with special needs. Each chapter has a multitude of informed contributors and is eloquently and concisely written. There are extensive references at the end of each chapter providing resources for those who may want more insight into certain topics. Congratulations to Marc B. Taub, Mary Bartuccio and Dominick M. Maino for providing our profession with such an outstanding and informative resource.

\section{Endnotes}

1 http://www.marchofdimes.com/baby/ loss_neonataldeath.html 